\title{
Chatting with the experts: exploring Irish consumer acceptance of new food technologies
}

\section{Gráinne Greehy}

\author{
Department of Food Business and Development, UCC
}

\section{Introduction}

As consumers, we often wonder about how the food we buy is produced and processed. For example, what ingredients are included or the impact of consuming such products on our health, or family's health and on society? Imagine coming across two boxes of apples in a supermarket; the apples in one box being labelled as 'genetically modified' and in the other box as 'GM free'. Which apple would you choose to purchase? Would the presence of the GM label impact your decision? Would being informed that the 'GM apple' was cheaper or healthier influence your judgment? These are the type of questions that my research addresses.

Government policy aims to develop Ireland as a knowledge-based bioeconomy and, as a result, has invested significantly in public and private research and development. Many of the outputs of this investment are new food technologies (NFTs) which are scientific and technological developments that may be adopted by industry to enhance the way food is produced or processed. Every food technology was considered 'new' at some point in time. For example, pasteurising milk was considered a radical food technology when first introduced a hundred years ago.

These technologies can provide the food industry with opportunities to gain a competitive advantage by satisfying consumers' diverse and increasingly conflicting demands from foods, including demands for convenient, tasty, healthy and affordable food products. Consumers may be more aware of some NFTs than others. For example, they may be familiar with genetically modifying food (which involves modifying an organism's genetic material using geneticengineering techniques.). However, they may not have heard about using nanotechnology in food production (which involves manipulating ingredients or packaging material at such a small scale it is invisible to the human eye). Equally, consumers may consider some NFTs to be more controversial than others. For example, they may have strong opinions about genetically modifying crops to increase their resistance to herbicides (i.e., weed killer). In contrast, consumers may be less opinionated about functional food products, such as probiotic yogurts, that claim to improve gut health. 


\section{Why consumer acceptance is important}

Investing in NFTs is futile if consumers proceed to reject these technologies. As is clearly evident from public negative reactions towards GM foods in the past, consumer acceptance of NFTs cannot be assumed and lack of acceptance can result in significant financial losses. The Government views the Irish food industry as part of the solution to the current recession. This solution will require investment in cutting edge technologies that enhance industry competitiveness. Given the scale of investment required to develop these technologies, it is important to determine which NFTs consumers would welcome and should, therefore, be developed and receive funding

It is widely acknowledged that consumers may evaluate and perceive these technologies differently to scientists and regulators. Therefore, there is now an appreciation of the need to engage with consumers and incorporate their opinions about these technologies. This engagement may also enable consumers to make more informed decisions about these technologies and potentially lead to greater consumer acceptance.

\section{Objectives of my research}

This research explores what Irish consumers think about NFTs, whether they would be willing to accept them and what factors influence their attitudes towards them. In addition, this research explores the impact of new information and engagement with a scientist on consumers' attitudes and acceptance.

The specific objectives of my research are:

To determine how acceptable consumers consider specific NFTs (and their different applications) to be, and how consumers' awareness and attitudes influence acceptance.

To explore the subjective values, including perceived risks, benefits and associated trade-offs, that influence (frame) consumers' attitudes and acceptance of NFTs.

To assess the overall influence of engaging with a scientist and new information on consumers' attitudes and acceptance of NFTs.

To identify what information causes shifts in consumer acceptance (i.e., potential 'tipping points').

Figure 1 illustrates the potential risk and benefit trade-offs that consumers could associate with NFTs. This figure draws attention to how the weighting of these risks and benefits by consumers could influence overall acceptance (rejection) of a technology. 


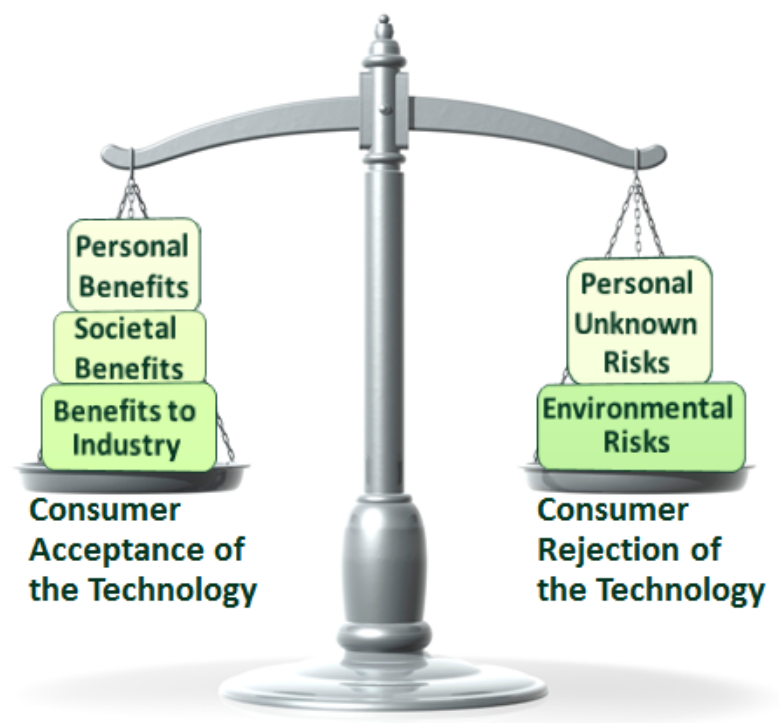

Figure 1: Potential Risk-Benefit Trade-Offs of a New Food Technology

The European Commission [The MASIS Report: Challenging Futures of Science in Society: Emerging Trends and Cutting-Edge Issues, 2009, p.17] has highlighted the need to engage with citizens in terms of scientific developments and "to experiment with ways of interaction, and evaluate where they might lead". Stage 1 of this research takes these recommendations to engage with the public into account by using a deliberative discourse (structured conversation) between food scientists and consumers for a variety of NFTs to allow for an unfolding of individual consumers' perspectives as information is presented to them. This approach provided depth rather than breadth in terms of examining consumers' attitudes.

\section{Methodology}

Four NFTs have been selected to be examined (as outlined in Figure 2) based on their numerous applications, varying degrees of novelty, associated moral and ethical concerns, proximity to market and regulatory issues.

Stage 1 of this research involved observations of a one-to-one deliberative discourse (structured conversation) between food scientists and consumers. During this conversation, the scientist discussed one of the four technologies and its (potential) food applications using simple everyday language. The scientist presented scenarios of different ways in which the technology could be applied during food production, to establish consumers' reactions towards such information. These scenarios illustrated hypothetical benefits and risks of different food applications of the technology from a consumer, societal, environmental and industry perspective. Video recording captured participants' non-verbal cues during 


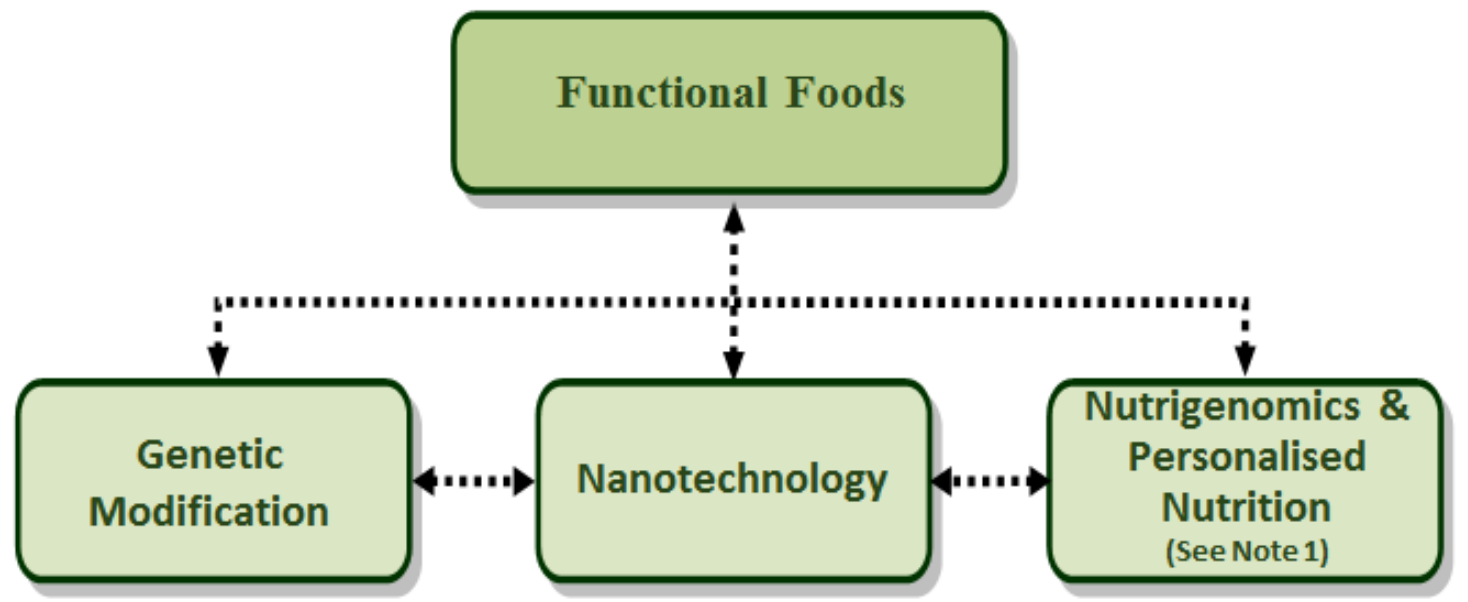

Figure 2: New Food Technologies to be Examined, both Individually (Stage 1) and Collectively (Stage 2)

the discourse that would not have been evident through audio recording alone. Furthermore, pre and post-discourse interviews with participants determined the influence of the discourse on consumers' acceptance and the factors contributing to any attitudinal change. The consumers also completed a brief questionnaire; the findings of which were compared to the qualitative findings.

\section{Findings to date and next steps}

The deliberative discourses proved useful in revealing the multiple factors influencing consumers' attitudes, and acceptance and how consumers constructed meaning and interpreted information about the technology in question. Generally, new information positively impacted consumers' attitudes and increased their likelihood, to varying degrees, of purchasing foods produced using the specific technology. Consumers either 'strongly approved' or 'somewhat approved' of the technology after participating in the discourse. However, other variables may have contributed to consumers' positive attitudes, including the information the scientist presented and interpersonal dynamics (e.g., trust and rapport) between participants.

Consumers were more accepting of the technology if they perceived associated personal and societal benefits to outweigh potential risks. Where consumers considered there to be a direct benefit to them personally or their families (e.g., a health benefit) or to society (e.g., an environmental benefit), they were more positive about the technology. However, if they perceived the technology to create negative consequences (e.g., potential risks to human health), they were reluctant to accept it. The extent of their reluctance depended on the consumers' individual characteristics (e.g., their risk sensitivity and atti- 
tude towards technological progress) and also their trust in stakeholders, including food producers and regulators. Finally, consumers' perceptions varied in terms of the applications, benefits and risks presented. For example, some consumers were more in favour of genetically modifying animals using animal genes whereas others preferred the concept of using plant genes.

Building on the findings from the deliberative discourses, Stage 2 will adopt a more traditional research approach. It will use in-depth interviews and a laddering technique, based on means-end chain (MEC) theory, to examine the factors influencing consumer acceptance of hypothetical functional food products produced using combinations of the other technologies. MEC is a framework for understanding the associations consumers make between product attributes and personally relevant and abstract consequences and values. A laddering technique enables an understating of what meanings consumers attach to different characteristics of a product.

Note 1: Nutrigenomics is the study of individuals' nutrient-gene interaction. Its practical applications involve the use of genetic testing for predisposition to diseases that can be mitigated with dietary interventions. It could potentially lead to the development of personalised nutrition which could involve segmenting the population based on pre-disposed illnesses and developing food products based on these profiles.

\section{Conclusions}

This research will contribute to studies completed to date on the factors influencing consumers' attitudes and acceptance of the four selected NFTs. While this research is exploratory in nature, it will provide industry, policymakers and government research agencies with information regarding consumer acceptance, attitude formation and risk perceptions, which can support the promotion and commercialisation of these technologies. Furthermore, this research will suggest some suitable messages and approaches for industry and institutions involved in food risk communications to provide consumers with information about NFTs to aid their evaluations of such technologies. Finally, this research will contribute to the academic debate on the differences between scientists' and lay public's formations of risk perceptions.

Gráinne Greehy is a researcher and 2nd year PhD student in the Department of Food Business and Development under the supervision of Dr. Mary McCarthy. The author wishes to thank her supervisor for her on-going guidance and support and her colleagues in Teagasc and the participating scientists and consumers for their contribution to this research. The author wishes to acknowledge funding received from the Department of Agriculture, Fisheries and Food, under the Food Institute Research Measure (FIRM) initiative to carry out this research. 